\title{
MALAT1 promotes angiogenesis of breast cancer
}

\author{
XIAO-JUAN HUANG ${ }^{1 *}$, YU XIA $^{1 *}$, GUI-FANG HE ${ }^{1}$, LU-LU ZHENG ${ }^{1}$, \\ YONG-PING CAI ${ }^{1,2}$, YU YIN ${ }^{1,2}$ and QIANG WU ${ }^{1,2}$ \\ ${ }^{1}$ Department of Pathology, Anhui Medical University, Hefei, Anhui 230032; ${ }^{2}$ Department of Pathology, \\ The First Affiliated Hospital of Anhui Medical University, Hefei, Anhui 230022, P.R. China
}

Received May 3,2018; Accepted September 3, 2018

DOI: $10.3892 /$ or.2018.6705

\begin{abstract}
Metastasis-associated lung adenocarcinoma transcript 1 (MALAT1) is a long non-coding RNA (lncRNA) that has an oncogenic role in some types of cancers, uncluding breast cancer (BC). To investigate the role of MALAT1 in human BC progression, we detected MALAT1 expression levels based on tissue samples from 20 BC cases and 20 healthy controls and found MALAT1 expression levels to be significantly high $(\mathrm{P}<0.05)$. Then, we knocked down endogenous MALAT1 in MCF-7 cells using MALAT1 short hairpin RNA (shRNA). The results revealed that MALAT1 knockdown could significantly inhibit proliferation, migration, and tube formation in vitro. In addition, miR-145 expression inversely changed in BC tissue cases. Furthermore, knockdown of endogenous MALAT1 significantly increased miR-145 levels in MCF-7 cells. This finding indicated an interaction between MALAT1 and miR-145. In addition, knockdown of MALAT1 significantly reduced the expression of vascular endothelial growth factor in MCF-7 cells. This outcome revealed that MALAT1 promoted angiogenesis in $\mathrm{BC}$, which may be related to the expression of miR-145.
\end{abstract}

\section{Introduction}

A long non-coding RNA (lncRNA) is a genomic transcript that is greater than 200 nucleotides and does not have a protein-coding function $(1,2)$. Emerging evidence has revealed that it is an important molecule for normal development and tumorigenesis (3-5). Moreover, recent studies have revealed that lncRNAs are involved in transcriptional, post-transcriptional, and epigenetic gene regulation $(6,7)$. Furthermore, numerous lncRNAs can function as oncogenes

Correspondence to: Dr Yu Yin or Dr Qiang Wu, Department of Pathology, Anhui Medical University, 81 Meishan Road, Hefei, Anhui 230032, P.R. China

E-mail: aydyinyu@aliyun.com

E-mail: aydjohn@yahoo.com

*Contributed equally

Key words: MALAT1, breast cancer, VEGF, miR-145, angiogenesis or tumor suppressors $(8,9)$. Metastasis-associated lung adenocarcinoma transcript 1 (MALAT1) is a large number of expressed lncRNAs, $~ 8,000$ nucleotides in length, identified as the first lncRNA associated with metastasis and survival of non-small cell carcinoma (7). In the absence of an open reading frame, MALAT1 exhibits no protein expression in in vitro translation (1). MALAT1 regulates gene expression and post-transcriptionally modifies primary transcripts, which are highly conserved among mammals (10). Aberrant expression levels of MALAT1 have been observed in various human malignancies, including lung, breast and bladder cancer as well as in osteosarcoma, colorectal cancer, and hepatocellular carcinoma (11-16).

MicroRNA-145 (miR-145), an important microRNA, is underexpressed and acts as a tumor-suppressor in several tumors. In addition, miR-145 has been revealed to inhibit proliferation and migration of breast cancer (BC) cells (17). miR-145 was notably downregulated in 106 pairs of normal and BC cancer tissues, and miR-145 played an inhibitory role in tumor angiogenesis, cell growth and invasion. Tumor growth takes place via the post-transcriptional regulation of new targets N-RAS and VEGF-A (18). miR-145 was revealed to be significantly downregulated in the plasma and cancer tissues of patients with colorectal cancer, and overexpression of miR-145 hindered cell proliferation, migration, and invasion (19). miR-145 was demonstrated to inhibit invasion and metastasis in osteosarcoma cells, mainly by downregulating the expression of vascular endothelial growth factor (VEGF) by specifically combining the $3^{\prime}$ untranslated region (3'UTR) of VEGF mRNA (20). In the present study, we investigated the following: i) MALAT1 expression in BC tissues; ii) the role of MALAT1 in the regulation of microvascular endothelial cell proliferation, migration, and tube formation of $\mathrm{BC}$ in vitro using the RNA interference approach; iii) the effect of MALAT1 on angiogenesis by VEGF; and iv) preliminary discussion of whether MALAT1 expression levels are correlated with miR-145 in BC.

\section{Materials and methods}

Tissue samples. Twenty pairs of invasive ductal carcinoma specimens and adjacent normal tissues were collected from the Department of Pathology of The First Affiliated Hospital of Anhui Medical University from January 2014 to December 2014, with the consent of patients and approval by the Ethics 
Committee of Anhui Medical University (Hefei, China). Surgical excised tumor samples were collected and rapidly stored in liquid nitrogen. Patients who had not received chemotherapy before surgery were selected. Samples were classified and selected according to the diagnosis using the anatomical pathology system (LOGENE pathology management software). Tissues were histologically and tumor cytologically evaluated prior to RNA sample extraction.

$R N A$ isolation, reverse transcription, and real-time quantitative polymerase chain reaction $(R T-q P C R)$. Tissue total RNA was extracted using TRIzol reagent according to the manufacturer's instructions (Invitrogen; Thermo Fisher Scientific, Inc., Waltham, MA, USA). Primers were designed and synthesized based on the gene sequences of MALAT1 and GAPDH, respectively. The MALAT1 primer sequences were as follows: Forward primer, 5'-AAAGCAAGGTCTCCCCAC AAG-3' and reverse primer, 5'-GGTCTGTGCTAGATCAAA AGGCA-3'. The GAPDH primers were as follows: Forward primer, 5'-ACCACAGTCCATGCCATCAC-3' and reverse primer, 5'-TCCACCCTGTTGCTGTA-3'. Total RNA $(1 \mu \mathrm{g})$ was reverse-transcribed into cDNA using the Transcriptor First Strand cDNA Synthesis Kit (Roche, Shanghai, China). One $\mu \mathrm{g}$ of the reverse transcription reaction solution was used as a template for fluorescence quantification and the relative levels of MALAT1 mRNA to control [glyceraldehyde 3-phosphate dehydrogenase (GAPDH)] transcripts were determined by RT-qPCR using the ABI 7500 Fast Real-Time PCR System. RT-qPCR amplification was performed in triplicate with reaction conditions beginning at $95^{\circ} \mathrm{C}$ for $10 \mathrm{~min}$, then $95^{\circ} \mathrm{C}$ for $10 \mathrm{sec}$ and $60^{\circ} \mathrm{C}$ for $30 \mathrm{sec}$ for 40 cycles. The relative expression level of MALAT1 was determined by the $2^{-\Delta \Delta \mathrm{Ct}}$ method (21).

Cell culture. Human BC cell lines (MCF-7) and human vascular endothelial cells (HUVECs) were provided by the Chinese Academy of Sciences (Shanghai, China). Human MCF-7 adenocarcinoma cells were cultured in Eagle's Minimum Essential Medium, and HUVECs were cultured in Endothelial Basal Medium (EBM2) (both from Lonza Group, Ltd. (Basel, Switzerland) complete medium with $0.5 \mathrm{ng} / \mathrm{ml}$ long-R3-IGF-1 (T\&L Biological Technology, Beijing, China), $0.1 \mathrm{mg} / \mathrm{ml}$ heparin and $10 \%$ fetal bovine serum (FBS). All cells were cultured in a humidified atmosphere with $5 \% \mathrm{CO}_{2}$ at $37^{\circ} \mathrm{C}$.

Design short hairpin RNA constructs and cell transfection. Lentivirus-encoded short hairpin RNA (shRNA) was provided by Shanghai GeneChem Co., Ltd. (Shanghai, China) and MALAT1 was knocked down. shRNA was cloned into pLKO.1 (GV248) lentiviral vectors. Targeted sequences of shMALAT1 and shNC were designed as follows: GGGCTT CTCTTAACATTTA and TTCTCCGAACGTGTCACGT, respectively. The BLAST search at www.ncbi.nih.nlm.gov was used to confirm the specificity of the shRNA sequence. The shRNA lentiviral infection construct was transfected into the MCF-7 cell lines with Polybrene. After two weeks of growth in $2 \mathrm{mg} / \mathrm{ml}$ puromycin, they were screened for a stable cell line and subsequently we determined the efficiency of MALAT1 knockdown by quantitative real-time PCR (RT-qPCR).

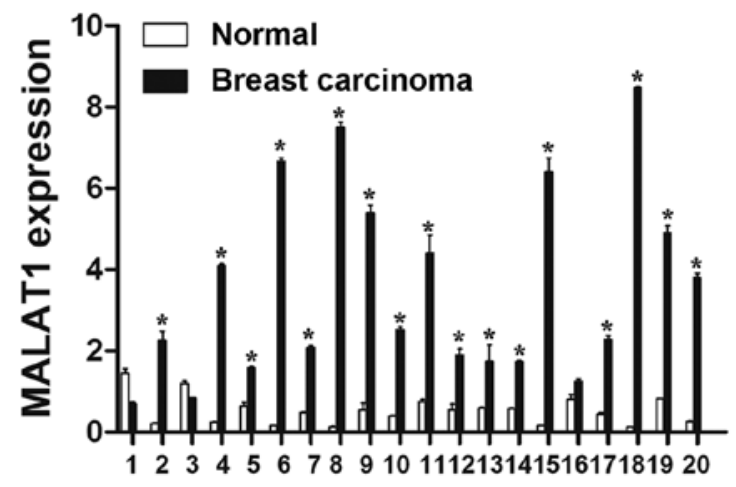

Figure 1. Expression of MALAT1 in breast cancer tissues. RT-qPCR analysis of relative expression levels of MALAT1 in 20 paired samples of breast cancer and adjacent non-cancerous tissues. RT-qPCR amplification was performed in triplicate, and the levels of MALAT1 were normalized against control GAPDH expression. Values are presented as the mean $\pm \mathrm{SD}$. ${ }^{*} \mathrm{P}<0.05$. MALAT1, metastasis-associated lung adenocarcinoma transcript 1.

RT-qPCR analysis of MALAT1, miR-145, and VEGF expression. Samples were ground and total RNA in tumor and normal tissues was extracted using TRIzol (Invitrogen; Thermo Fisher Scientific, Inc.). Reverse transcription of cDNA was performed using the PrimeScript ${ }^{\circledR}$ RT reagent kit (Takara Biotechnology Co., Ltd., Dalian, China). The designed primers were as follows: MALAT1: Forward, 5'-AAAGCAAGGTCT CCCCACAAG-3' and reverse, 5'-GGTCTGTGCTAGATC AAAAGGCA-3'. MALAT1 expression was quantified by ABI PRISM 7500 system (Applied Biosystems; Thermo Fisher Scientific, Inc.) using the SYBR ${ }^{\circledR}$ Premix DimerEraser Kit (Takara Biotechnology Co., Ltd.). GAPDH was used as a control. The primers for GAPDH were as follows: Forward, 5'-ACCACAGTCCATGCCATCAC-3' and reverse, 5'-TCC ACCCTGTTGCTGTA-3'. RT-qPCR analysis of miR-145 was performed using miRNeasy Kit, and cDNA was reversely transcribed using the miScript ${ }^{\circledR}$ II RT kit and miScript ${ }^{\circledR}$ $\mathrm{SYBR}^{\circledR}$-Green PCR kit (all from Qiagen, Inc., Valencia, CA, USA) used with miScript miRNA PCR Arrays. U6 snRNA was used as the endogenous control. The cycling program was performed using ABI PRISM 7500 (Applied Biosystems; Thermo Fisher Scientific, Inc.). The procedure and conditions for PCR were: $95^{\circ} \mathrm{C}$ for $2 \mathrm{~min}$ and $30 \mathrm{sec}$, followed by 35 cycles at $94^{\circ} \mathrm{C}$ for $30 \mathrm{sec}, 62^{\circ} \mathrm{C}$ for $30 \mathrm{sec}$, and $72^{\circ} \mathrm{C}$ for $1 \mathrm{~min}$ and $20 \mathrm{sec}$. Each experiment was repeated three times and the expression of MALAT1, miR-145 and VEGF was quantified using the $2^{-\Delta \Delta \mathrm{Ct}}$ method (21).

Cell viability assay. The influence of shMALAT1 on the proliferation of HUVECs was demonstrated using cell viability assays. BC cells were transfected into $4 \times 10^{3} \mathrm{HUVEC} /$ well in 96-well culture plates. The shNC from non-transfected cells were used as controls. Cell viability was assessed for proliferation at 24, 48, 72, 96 and 120 h by Cell Counting Kit-8 (CCK-8) (Dojindo Molecular Technologies, Inc., Kumamoto, Japan) according to the manufacturer's instructions. Results were obtained in three independent experiments, and each experiment was repeated three times and expressed as the means \pm standard deviation. Then, the absorbance at $570 \mathrm{~nm}$ was detected on a microplate reader. 


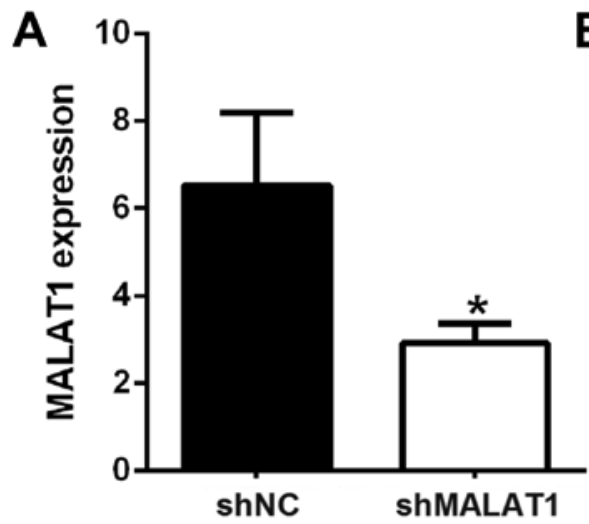

B
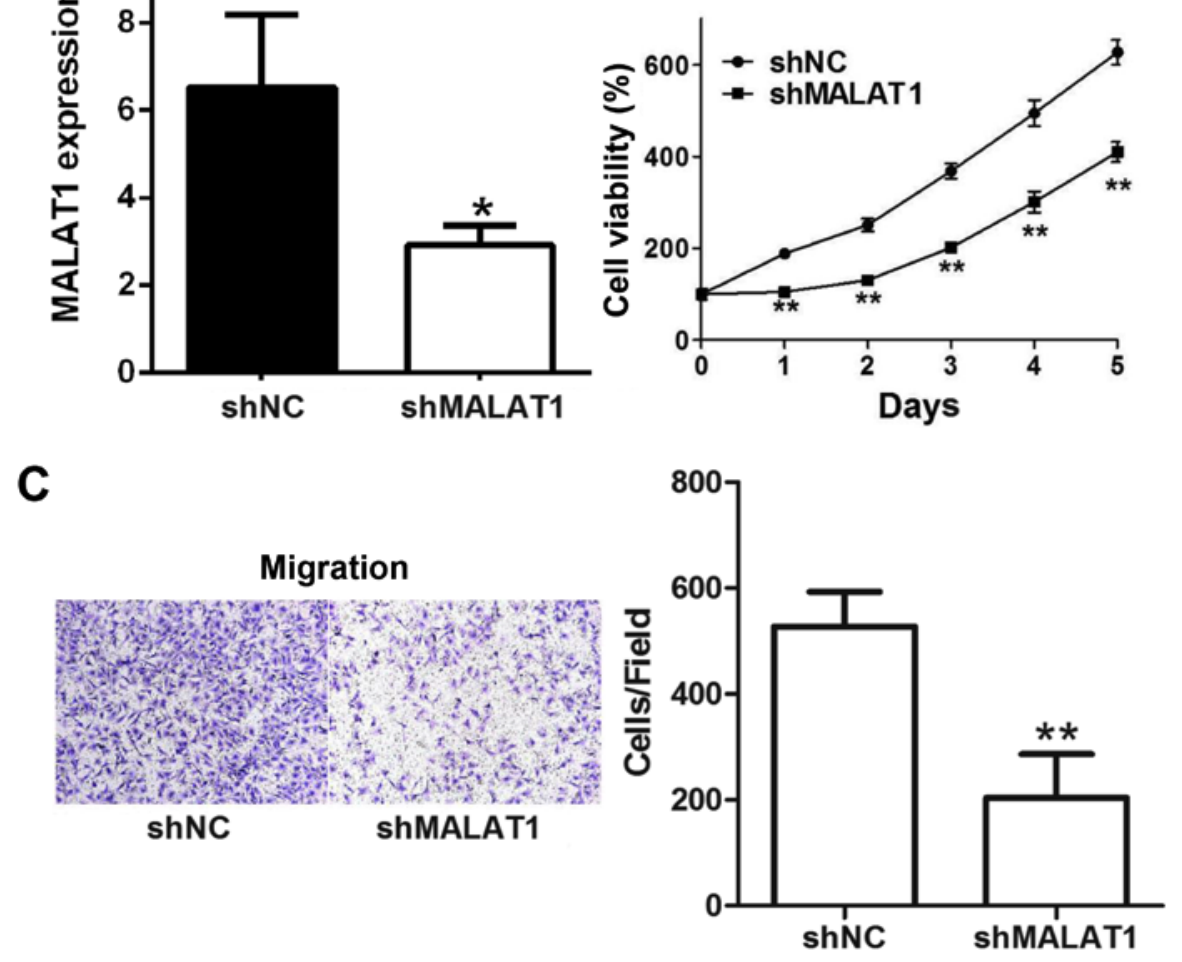

D
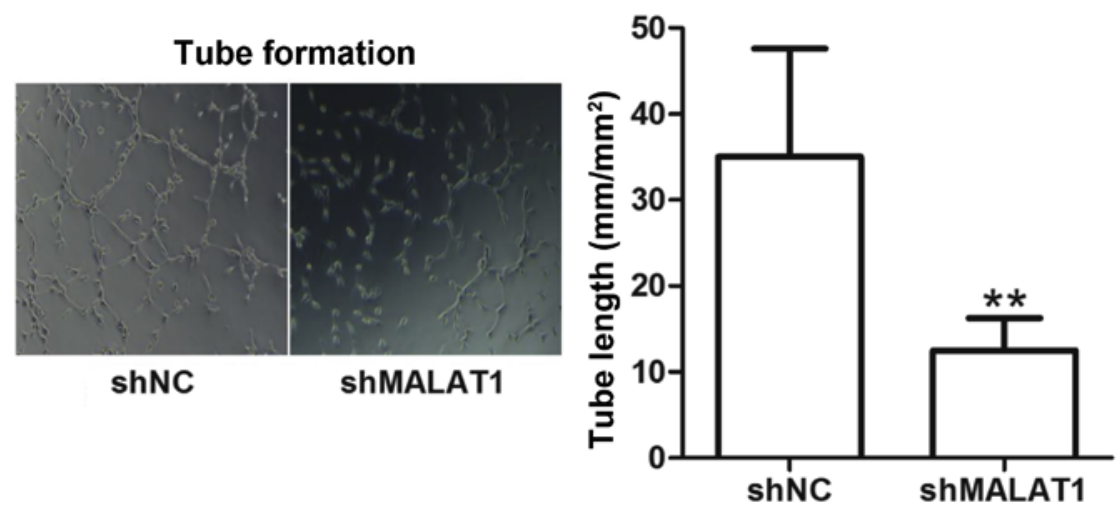

Figure 2. Downregulation of MALAT1 in breast cancer cell microvascular endothelial cells significantly inhibits proliferation, migration, and tube formation in vitro. (A) qPCR analysis of relative expression levels of MALAT1 in MCF-7 cells and levels of MALAT1 were normalized against control GAPDH expression. Results were obtained from triple replicates and are presented as mean $\pm \mathrm{SD}$. ${ }^{*} \mathrm{P}<0.05$. (B) Knockdown of MALAT1 expression on the proliferation of endothelial cells. Cell viability was assessed for proliferation using a cell-activated CCK-8 kit. The results revealed that the activity of the endothelial cells in the shMALAT1 group was significantly lower than that in the shNC group. Results were obtained from triple replicates and are presented as the mean \pm SD. ${ }^{* *} \mathrm{P}<0.01$. (C) Effects of knockdown of MALAT1 on the migration abilities of HUVECs, and HUVEC migration assays were performed through co-culturing transfected cells with HUVECs in 24-well plates with Transwell chambers. Cell migration was assessed at $18 \mathrm{~h}$ in a Transwell assay in a blinded manner Values are presented as the mean $\pm \mathrm{SD}$. ${ }^{* *} \mathrm{P}<0.01$. (D) Tube formation assays. Cells were seeded on Matrigel-coated wells in the presence of different $\mathrm{CM}$, as indicated, and incubated for $18 \mathrm{~h}$ to form a capillary network. The total number of branched tubes was then counted. Values are presented as the mean \pm SD ${ }^{* *} \mathrm{P}<0.01$ ( $\mathrm{n}=3$, Student's t-test). MALAT1, metastasis-associated lung adenocarcinoma transcript 1; CCK-8, Cell Counting Kit-8.

Migration assay. MCF-7 cells were seeded at a density of $1 \times 10^{5}$ cells/well in 24 -well culture plates. Twenty-four hours later, shMALAT1 and shNC vector plasmids were transfected into MCF-7 cells using (Invitrogen; Thermo Fisher Scientific, Inc.) according to the manufacturer's protocol. After $24 \mathrm{~h}$, the Transwell chamber was placed in 24-well plates containing transfected cells. Then, $1 \times 10^{5}$ HUVECs were added to the upper chamber with a cell suspension volume of $200 \mu \mathrm{l}$, placed in $5 \% \mathrm{CO}_{2}$ at $37^{\circ} \mathrm{C}$ for $18 \mathrm{~h}$. Media (Endothelial Basel Medium complete medium with $0.5 \mathrm{ng} / \mathrm{ml}$ long-R3-IGF-1, $0.1 \mathrm{mg} / \mathrm{ml}$ heparin and 10\% FBS) containing transfected cells in the lower chamber were used as chemoattractants. Subsequently, the upper chamber liquid and cells were removed, and the number of cells in the lower chamber were stained with $0.1 \%$ crystal violet for $30 \mathrm{~min}$, and then observed with an inverted microscope after the removal of the microporous membrane. 
The number of migrated cells were counted using a 40X objective and images were captured randomly at five different fields of view. Each experiment was repeated three times.

Assays for tube formation of HUVECs. After the Matrigel had thawed at $4^{\circ} \mathrm{C}, 50 \mu 1$ Matrigel Basement Membrane Matrix (BD Biosciences, Franklin Lakes, NJ, USA) was added to each well of 96-well cell culture plates, and the endothelial cells were trypsinized into a single-cell suspension using endothelial cell culture medium. The cells were counted and seeded in 96-well cell culture plates pre-coated with Matrigel at a concentration of 1,500 cells/well. After culturing for 24 h, 6 fields (magnification, x40) were randomly selected and images were captured using an inverted microscope The length of microvessel branches and the number of branches were counted. The experiment was repeated three times.

Statistical analysis. Data were presented as the mean $\pm \mathrm{SD}$ using SPSS 17.0 (SPSS, Inc., Chicago, IL, USA) for data analysis and repeated at least three times. Quantitative data were compared using Student's t-test. Correlation analysis was performed using Spearman's rank test. $\mathrm{P}<0.05$ was considered to indicate a statistically significant difference, and * and ** symbols indicated the significance of 0.05 and 0.01 levels, respectively.

\section{Results}

Upregulation of MALAT1 expression in human breast cancer tumor tissues. In a collection of 20 pairs of randomly selected samples from BC patients, the MALAT1 expression level was significantly higher in tumor tissues compared with adjacent non-cancerous tissues $(\mathrm{P}<0.05)$ (Fig. 1).

Downregulation of MALAT1 in BC microvascular endothelial cells significantly inhibit proliferation, migration, and tube formation in vitro. To investigate the biological significance of MALAT1 on MCF-7 cells, we first knocked down endogenous MALAT1 in MCF-7 cells using MALAT1 short hairpin RNA (shRNA). Knockdown of MALAT1 significantly decreased the expression of MALAT1 MCF-7 cells. This result indicated that shMALAT1 was successfully transfected in MCF-7 cells $(\mathrm{P}<0.05)$ (Fig. 2A).

The effect of knockdown of MALAT1 expression on the proliferation of endothelial cells was confirmed by cell-activated CCK-8 assay. The results revealed that the activity of endothelial cells in the shMALAT1 group was significantly lower than that in the shNC group $(\mathrm{P}<0.01)$ (Fig. 2B).

Transwell migration assays were used to assess the migration abilities of endothelial cells with MALAT1 knockdown. The rate of endothelial cells in the shMALAT1 group was significantly lower than that in the shNC group $(\mathrm{P}<0.01)$ (Fig. $2 \mathrm{C})$.

Assays for tube formation were perfomed. Upon observation compared with the shNC group, tubular structures and tubular length decreased in the shMALAT1 group $(\mathrm{P}<0.01)$ (Fig. 2D), indicating that shMALAT1 can significantly curb the proliferation, migration, and tube formation of HUVECs in vitro.

Knockdown of MALAT1 reduces VEGF expression in MCF-7 cells. To further research the potential mechanism of subtractive

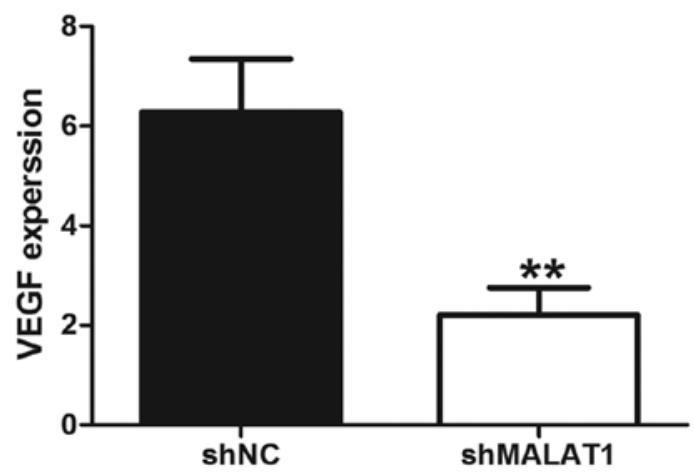

Figure 3. Downregulation of MALAT1 in breast cancer cells. qPCR analysis of the relative expression level of VEGF in MCF-7 cells and the levels of VEGF were normalized against control GAPDH expression. Amplification was performed in triplicate. ${ }^{* *} \mathrm{P}<0.01$. MALAT1, metastasis-associated lung adenocarcinoma transcript 1; VEGF, vascular endothelial growth factor.

regulation of MALAT1, we assessed the expression of VEGF. Knockdown of MALAT1 significantly reduced the expression level VEGF (P<0.01) (Fig. 3).

Downregulation of miR-145 expression in human BC tumor tissues. To evaluate the functional relative relevance of MALAT1 and miR-145 in BC, the MALAT1 and miR-145 expression levels were assessed in clinical specimens by RT-qPCR. The expression level of miR-145 was significantly lower in $\mathrm{BC}$ tissues than that in adjacent non-cancerous tissues $(\mathrm{P}<0.01)$ (Fig. 4A). Quantitative and regression analysis of MALAT1 and miR-145 expression in the 20 cancer cases revealed that there was a negative correlation between miR-145 and MALAT1 expression in BC tissues $\left(r_{s}=-0.6782\right.$, $\mathrm{P}=0.001$ ) (Fig. 4B).

Knockdown of endogenous MALAT1 also significantly increases the expression level of miR-145 in MCF-7 cells. We searched the online bioinformatics database (starBase, v2.0, http://starbase.sysu.edu.cn/) and found that miR-145 and MALAT1 have specific binding sites (Fig. 5A). To further confirm the reciprocal effect between miR-145 and MALAT1, we knocked down endogenous MALAT1 in MCF-7 cells using MALAT1 shRNA. Knockdown of endogenous MALAT1 significantly increased the expression level of miR-145 in MCF-7 cells $(\mathrm{P}<0.01)$ (Fig. 5B). This result indicated a mutual interaction between MALAT1 and miR-145.

\section{Discussion}

Breast cancer (BC) is a common cancer in women worldwide and has the second highest mortality rate after lung cancer (22). Evidence suggests that in addition to short microRNAs, lncRNA transcripts of 200 -nt in length do not encode proteins, but affect tumorigenesis by modulating the expression levels of coding genes. They are valuable biomarkers and therapeutic targets due to their role in the necessary pathways for the initiation and progression of tumors, combined with their tissue and stage specificity (23-25). Studies have revealed the overexpression of MALAT1 in hepatocellular carcinoma and uterine endometrial stromal sarcoma $(16,26)$. MALAT1 
A

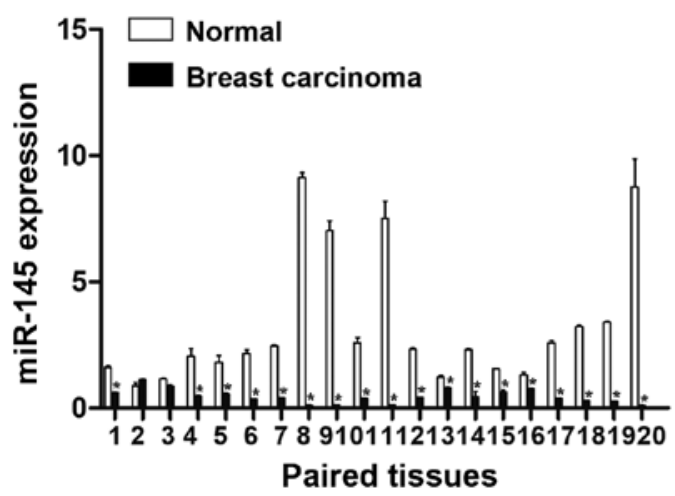

B

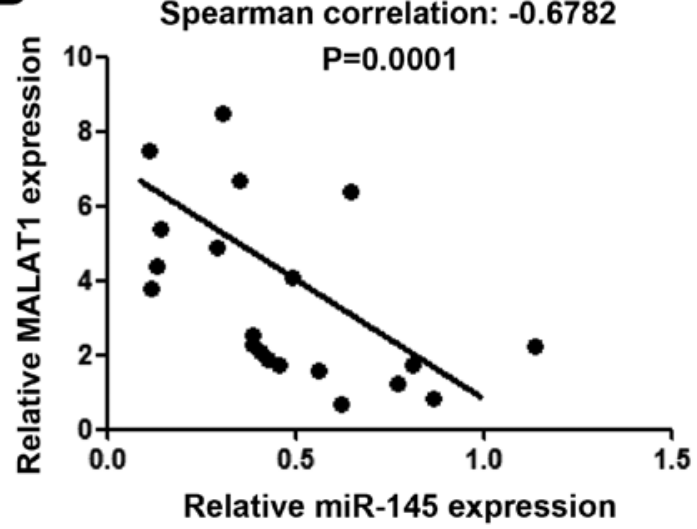

Figure 4. Expression of miR-145 in breast cancer tissues. (A) RT-qPCR analysis of the relative expression levels of miR-145 in breast cancer tissues. U6 small nuclear RNA levels were used as an internal control. Values are presented as the mean \pm SD. ${ }^{*}<<0.05$. (B) Linear regression analysis of MALAT1 and miR-145 expression in breast cancer tissues $(n=20)$. MALAT1, metastasis-associated lung adenocarcinoma transcript 1.

A

MALAT1 5' AAGTTTGAAGTGGAAAACTGGAA-3'

||||||||||

miR-145 3'-TCCCTAAGGACCCTTTTGACCTG-5
B

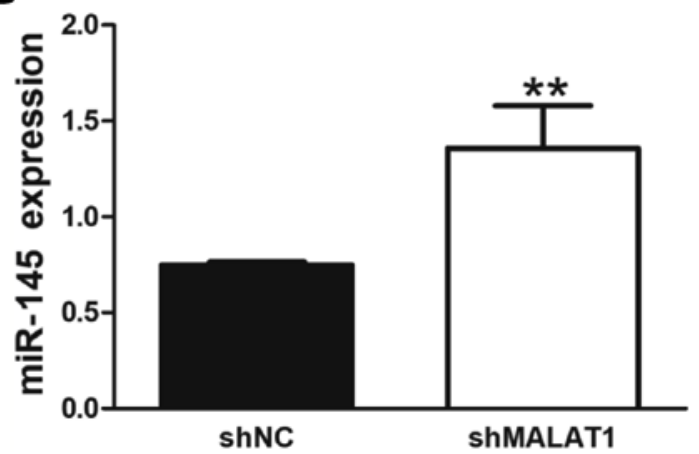

Figure 5. Downregulation of MALAT1 in breast cancer cells. (A) Predicted binding sites between MALAT1 and miR-145. (B) qPCR analysis of the relative expression levels of miR-145 in MCF-7 cells and the levels of MALAT1 were normalized against control U6 expression. Amplification was performed in triplicate. ${ }^{* *} \mathrm{P}<0.01$. MALAT1, metastasis-associated lung adenocarcinoma transcript 1.

plays an important role in the proliferation, migration, invasion, and metastasis of lung cancer (27). MALAT1 has also been reported as an important endogenous regulator of mesenchymal stem cell proliferation, angiogenesis, and immunosuppression (28). MALAT1 knockdown and proliferation of human choriocarcinoma cells in vitro were markedly hindered, and the tumor size in vivo was reduced. The study indicated that MALAT1 may be an oncogenic lncRNA promoting proliferation of choriocarcinoma and it may be a therapeutic target in human choriocarcinoma (29).

In our study, the expression level of MALAT1 was notably higher in tumor tissues than in adjacent non-cancerous tissues $(\mathrm{P}<0.01$, Fig. 1). To investigate the influence of MALAT1 on the proliferation, migration, and angiogenesis of BC microvascular endothelial cells in the present study, cells were transfected with shMALAT1 in vitro. Knockdown efficiency was evaluated by qPCR ( $\mathrm{P}<0.01$, Fig. $2 \mathrm{~A})$, and downregulation of endothelial cell MALAT1 expression notably hindered the proliferation of endothelial cells in vitro $(\mathrm{P}<0.01$, Fig. 2B) and migration ( $\mathrm{P}<0.01$, Fig. $2 \mathrm{C}$ ) while reducing the ability of in vitro tube formation $(\mathrm{P}<0.01$, Fig. $2 \mathrm{D})$. The above indicators were key indicators reflecting the functional activity of endothelial cells.The aforementioned experiments indicated that MALAT1 could regulate the biological activity of endothelial cells and inhibit vascular structure.

Angiogenesis in tumors refers to the process of producing new capillaries from existing capillaries, as opposed to the vasculogenesis of embryonic fibroblasts, wherein early endothelial cells differentiate into new blood vessels. Angiogenesis under physiological conditions is a strictly controlled process found only in the context of development, regeneration, and wound repair. Pathological angiogenesis is a continuous, noncontrolled process that can promote tumor growth, invasion, and metastasis. Without angiogenesis, tumors larger than 1-2 $\mathrm{mm}$ in diameter may fail to grow due to the lack of nutrients. Therefore, tumor angiogenesis is important in tumor growth and progression, whereas vascular endothelial growth of VEGF is the strongest angiogenic factor and is crucial in the formation of vascular systems, regulation of the progress and perviousness of vascular endothelial cells, and neovascularization of tumors (30). Studies have revealed that blocking VEGF signaling inhibits tumor angiogenesis, thus achieving tumor growth inhibition. As a central regulator of angiogenesis, VEGF is heavily targeted in anti-angiogenic therapies (31). Studies (32) have demonstrated that miRNAs are important in VEGF-mediated tumorigenesis and new angiogenesis. 
Our assays for tube formation revealed that compared with the shNC group, tubular structures and tubular length were decreased in the shMALAT1 group (P<0.01, Fig. 2D), inferring that shMALAT1 can significantly inhibit tube formation of HUVECs in vitro. To further research the potential mechanism of negative adjustment of MALAT1, we assessed VEGF expression. Knockdown of MALAT1 significantly reduced the expression level of VEGF $(\mathrm{P}<0.01)$ (Fig. 3). This result indicated that MALAT1 promotes BC angiogenesis.

In our study, the expression of miR-145 was downregulated in BC tissues (Fig. 4A), whereas MALAT1 was upregulated in BC tissues (Fig. 1) and the expression of miR-145 was negatively correlated with MALAT1 expression in BC tissues $\left(r_{s}=-0.6782, P=0.001\right.$, Fig. 4B). This finding indicated interaction between MALAT1 and miR-145. In 2006, transfecting synthetic RNA transcripts containing tandem miRNA binding sites into cells regulated the abundance of intracellular miRNAs (33). We also confirmed that the mRNA of the protein-encoding gene, pseudogenes, and lncRNAs can be used as miRNA molecular sponges that regulate the miRNA or miRNA expression of other target genes $(25,34)$. Researcher also confirmed that the mRNA of the protein-encoding gene, pseudogenes, and lncRNAs can be used as miRNA molecular sponges that play a significant role in regulating the miRNA or miRNA expression of other target genes $(25,34)$.We predicted miRNA targeting MALAT1 using http://www.mircode.org/ and revealed 105 binding sites on the MALAT1 RNA strand, downregulated expression of miR-145 in BC, and its presence in the MALAT1 RNA chain-binding site (Fig. 5A). The expression of miR-145 was also upregulated in BC cells by knocking down MALAT1 ( $\mathrm{P}<0.01$, Fig. 5B), suggesting interaction between MALAT1 and miR-145. However, the specific relationship warrants further study.

\section{Acknowledgements}

Not applicable.

\section{Funding}

The present study was supported in part by the National Natural Science Foundation of China (no. 81201536), the China Postdoctoral Science Foundation funded project (nos. 2016M590561 and 2017T100441), and the Postdoctoral Science Foundation funded project of Anhui Province (no. 2015B051).

\section{Availability of data and materials}

The datasets used or analyzed during the current study are available from the corresponding author on reasonable request.

\section{Authors' contributions}

YY, YPC and QW conceived and designed the study and the experiments. XJH, YX, GFH and LLZ performed the experiments. XJH and YX analyzed the data. GFH and LLZ collected the data. XJH wrote the manuscript. YY, YPC and QW reviewed and edited the manuscript. YY acquired funding. All authors have read and approved the final manuscript and agree to be accountable for all aspects of the research in ensuring that the accuracy or integrity of any part of the work are appropriately investigated and resolved.

\section{Ethics approval and consent to participate}

The study was approved by the Ethics Committee of Anhui Medical University (Hefei, China) and consent was obtained from patients.

\section{Patient consent for publication}

Not applicable.

\section{Competing interests}

There authors declare that they have no competing interests.

\section{References}

1. Ji P, Diederichs S, Wang W, Böing S, Metzger R, Schneider PM Tidow N, Brandt B, Buerger H, Bulk E, et al: MALAT-1, a novel non-coding RNA, and thymosin beta4 predict metastasis and survival in early-stage non-small cell lung cancer. Oncogene 22: 8031-8041, 2003

2. Tripathi V, Ellis JD, Shen Z, Song DY, Pan Q, Watt AT, Freier SM, Bennett CF, Sharma A, Bubulya PA, et al: The nuclear-retained non-coding RNA MALAT1 regulates alternative splicing by modulating SR splicing factor phosphorylation. Mol Cell 39: 925-938, 2010.

3. Tsai MC, Spitale RC and Chang HY: Long intergenic non-coding RNAs: New links in cancer progression. Cancer Res 71: 3-7, 2011.

4. Kornienko AE, Guenzl PM, Barlow DP and Pauler FM: Gene regulation by the act of long non-coding RNA transcription. BMC Biol 11: 59, 2013.

5. Xu S, Jin C, Shen X, Fang D, Zhu J and Fu G: MicroRNAs as potential novel therapeutic targets and tools for regulating paracrine function of endothelial progenitor cells. Med Sci Monit 18: HY27-HY31, 2012.

6. Mercer TR, Dinger ME and Mattick JS: Long non-coding RNAs: Insights into functions. Nat Rev Genet 10: 155-159, 2009.

7. Wilusz JE, Sunwoo H and Spector DL: Long non-coding RNAs: Functional surprises from the RNA world. Genes Dev 23: 1494-1504, 2009.

8. Li T, Xie J, Shen C, Cheng D, Shi Y, Wu Z, Deng X, Chen H, Shen B, Peng C, et al: Amplification of long non-coding RNA ZFAS1 promotes metastasis in hepatocellular carcinoma. Cancer Res 75: 3181-3191, 2015.

9. Liu B, Sun L, Liu Q, Gong C, Yao Y, Lv X, Lin L, Yao H, Su F, Li D, et al: A cytoplasmic NF-kappaB interacting long non-coding RNA blocks IkappaB phosphorylation and suppresses breast cancer metastasis. Cancer Cell 27: 370-381, 2015.

10. Schorderet P and Duboule D: Structural and functional differences in the long non-coding RNA hotair in mouse and human. PLoS Genet 7: e1002071, 2011.

11. Schmidt LH, Spieker T, Koschmieder S, Schäffers S, Humberg J, Jungen D, Bulk E, Hascher A, Wittmer D, Marra A, et al: The long non-coding MALAT-1 RNA indicates a poor prognosis in non-small cell lung cancer and induces migration and tumor growth. J Thorac Oncol 6: 1984-1992, 2011.

12. Fan L, Strasser-Weippl K, Li JJ, St Louis J, Finkelstein DM, Yu KD, Chen WQ, Shao ZM and Goss PE: Breast cancer in China. Lancet Oncol 15: e279-e289, 2014.

13. Ying L, Chen Q, Wang Y, Zhou Z, Huang Y and Qiu F: Upregulated MALAT-1 contributes to bladder cancer cell migration by inducing epithelial-to-mesenchymal transition. Mol Biosyst 8: 2289-2294, 2012.

14. Fellenberg J, Bernd L, Delling G, Witte D and ZahltenHinguranage A: Prognostic significance of drug-regulated genes in high-grade osteosarcoma. Mod Pathol 20: 1085-1094, 2007.

15. Xu C, Yang M, Tian J, Wang X and Li Z: MALAT-1: A long non-coding RNA and its important 3 ' end functional motif in colorectal cancer metastasis. Int J Oncol 39: 169-175, 2011. 
16. Lin R, Maeda S, Liu C, Karin M and Edgington TS: A large non-coding RNA is a marker for murine hepatocellular carcinomas and a spectrum of human carcinomas. Oncogene 26: 851-858, 2007.

17. Ding Y, Zhang C, Zhang J, Zhang N, Li T, Fang J, Zhang Y, Zuo F, Tao Z Tang S, et al: miR-145 inhibits proliferation and migration of breast cancer cells by directly or indirectly regulating TGF- $\beta 1$ expression. Int J Oncol 50: 1701-1710, 2017.

18. Zou C, Xu Q, Mao F, Li D, Bian C, Liu LZ, Jiang Y, Chen X, Qi Y, Zhang X, et al: MiR-145 inhibits tumor angiogenesis and growth by N-RAS and VEGF. Cell Cycle 11: 2137-2145, 2012.

19. Yin Y, Yan ZP, Lu NN, Xu Q, He J, Qian X, Yu J, Guan X Jiang BH and Liu LZ: Downregulation of miR-145 associated with cancer progression and VEGF transcriptional activation by targeting N-RAS and IRS1. Biochim Biophys Acta 1829 239-247, 2013

20. Fan L, Wu Q, Xing X, Wei Y and Shao Z: MicroRNA-145 targets vascular endothelial growth factor and inhibits invasion and metastasis of osteosarcoma cells. Acta Biochim Biophys Sin 44: 407-414, 2012.

21. Livak KJ and Schmittgen TD: Analysis of relative gene expression data using real-time quantitative PCR and the $2^{-\Delta \Delta C_{\mathrm{T}}}$ method. Methods 25: 402-408, 2001.

22. Mohinta S, Wu H, Chaurasia $\mathrm{P}$ and Watabe $\mathrm{K}$ : Wnt pathway and breast cancer. Front Biosci 12: 4020-4033, 2007.

23. Geng YJ, Xie SL, Li Q, Ma J and Wang GY: Large intervening non-coding RNA HOTAIR is associated with hepatocellular carcinoma progression. J Int Med Res 39: 2119-2128, 2011

24. Gutschner T, Baas M and Diederichs S: Non-coding RNA gene silencing through genomic integration of RNA destabilizing elements using zinc finger nucleases. Genome Res 21: 1944-1954, 2011.

25. Poliseno L, Salmena L, Zhang J, Carver B, Haveman WJ and Pandolfi PP: A coding-independent function of gene and pseudogene mRNAs regulates tumour biology. Nature 465: 1033-1038, 2010 .
26. Luo JH, Ren B, Keryanov S, Tseng GC, Rao UNM, Monga SP, Strom S, Demetris AJ, Nalesnik M, Yu YP, et al: Transcriptomic and genomic analysis of human hepatocellular carcinomas and hepatoblastomas. Hepatology 44: 1012-1024, 2006.

27. Hanahan D and Weinberg RA: Hallmarks of cancer: The next generation. Cell 144: 646-674, 2011.

28. Li X, Song Y, Liu F, Liu D, Miao H, Ren J, Xu J, Ding L, Hu Y, Wang Z, et al: Long non-coding RNA MALAT1 promotes proliferation, angiogenesis, and immunosuppressive properties of mesenchymal stem cells by inducing VEGF and IDO. J Cell Biochem 118: 2780-2791, 2017.

29. Shi D, Zhang Y, Lu R and Zhang Y: The long non-coding RNA MALAT1 interacted with miR-218 modulates choriocarcinoma growth by targeting Fbxw8. Biomed Pharmacother 97: 543-550, 2018.

30. Plate KH, Breier G, Weich HA and Risau W: Vascular endothelial growth factor is a potential tumour angiogenesis factor in human gliomas in vivo. Nature 359: 845-848, 1992.

31. Vlahakis NE, Young BA, Atakilit A, Hawkridge AE, Issaka RB, Boudreau N and Sheppard D: Integrin alpha9beta1 directly binds to vascular endothelial growth factor (VEGF)-A and contributes to VEGF-A-induced angiogenesis. J Biol Chem 282: 15187-15196, 2007.

32. Xu Q, Liu LZ, Qian X, Chen Q, Jiang Y, Li D, Lai L and Jiang BH: MiR-145 directly targets p70S6K1 in cancer cells to inhibit tumor growth and angiogenesis. Nucleic Acids Res 40: 761-774, 2012.

33. Ebert MS and Sharp PA: Emerging roles for natural MicroRNA sponges. Curr Biol 20: R858-R861, 2010.

34. Tay Y, Kats L, Salmena L, Weiss D, Tan SM, Ala U, Karreth F, Poliseno L, Provero P, Di Cunto F, et al: Coding-independent regulation of the tumor suppressor PTEN by competing endogenous mRNAs. Cell 147: 344-357, 2011 